\title{
Mapping diversity: from ecology and human geography to urbanism and culture
}

\author{
Dan C. Baciu ${ }^{1}$ (D) Duola $\mathrm{Mi}^{2}$ (D) $\cdot$ Callum Birchall ${ }^{1}$ (D) - Diana Della Pietra ${ }^{1}$ (D) \\ Luca van Loevezijn ${ }^{1}$ (D) Anna Nazou ${ }^{1}$ (D)
}

Received: 30 June 2021 / Accepted: 7 June 2022

(c) The Author(s) 2022

\begin{abstract}
A large body of research across science and humanities has come to deal with diversity, which, as a scientific concept, has proved immensely relevant in helping researchers understand anything from ecosystems and natural habitats to cities and culture. Here, we develop a first method to quantify and map urban diversity. Our article begins with a concrete example through which we demonstrate how to apply a basic version of our method to create a diversity map for a given urban area. This map is easy to interpret and can be used to accurately locate the most diverse centers of urban activity. We then go on to show how our basic method can be expanded to quantify many different types of urban diversity, and how it can be used to create regional and global diversity maps. Such diversity maps are relevant in both studying diversity and modeling the dynamics of diversification in urban environments. We conclude the article by making a bridge to other scientific disciplines, and by proposing six key steps that may serve as a foundation for a general framework for the evaluation and mapping of diversity across all fields of science.
\end{abstract}

Keywords Cultural life · Diversification cycles · Urban diversity · Urban diversification $\cdot$ Diversity index $\cdot$ Diversity mapping $\cdot$ Diversification mapping

\section{Introduction}

Many scientists and scholars, often working independently of one another, have come to recognize how immensely valuable the scientific concept of diversity is. The study of diversity allows researchers to discover and address real-world problems of utmost relevance. For example, most life scientists-if not all-have

\footnotetext{
Dan C. Baciu

books@yahoo.com

1 Delft University of Technology, Delft, The Netherlands

2 The University of Arizona, Tucson, USA
} 
come to agree that decaying biodiversity deeply threatens life on Earth (Wilson 1999; Schweiger and Laliberté 2022). Similarly, many humanities scholars who have witnessed the disappearance of entire languages and cultures, sometimes under severe oppression, advocate for respect toward cultural diversity (Wurm 1996).

The evaluation of diversity goes beyond life science and cultural studies. In environmental science, methods of mapping the diversity of soils have led to important developments in the field (Guo et al. 2003; Amundson 2021), and, in economics, the intricate evaluation of diversity is relevant for anything from portfolios and antitrust economics to economic bubbles and resilience (United Nations Climate Change 2021).

Given this great relevance of the concept of diversity across a broad array of sciences, it can only be expected that discoveries made in one discipline have been independently made in or have come to be translated to others. For example, ecologists have discovered that they can create global maps of diversity (Jenkins et al. 2013; Schweiger and Laliberté 2022), but such maps are also found in genetics (Miraldo et al. 2016) and soil science (Ibáńez et al. 1995), and diversity mapping has more recently entered human geography (Vertovec et al. 2021; Raszka 2022). Although diversity maps are found across multiple sciences, a more unified theoretical framework that unites the evaluation of diversity across all sciences has not yet been proposed.

We begin this present article by broadening the evaluation and mapping of diversity in two important directions: We study (1) urban diversity and (2) cultural diversity in urban space. By urban diversity, we mean in particular the diversity of urban activities and of architectural types and styles that are present in a given area of the city. By cultural diversity in urban space, we mean the diversity of ideas that entire collectives of people mentally associate with a given urban or architectural space.

These two new research directions, together with a brief review of previous work, lead us to propose a unified framework for the evaluation and mapping of diversity. All scientists - and their students, too-can use this framework to integrate their discoveries.

The article is structured as follows:

In the first section, "Mapping urban diversity: rationale and example," we demonstrate how to apply a simplified version of our framework to evaluate and map diversity in a given urban area. This section is a practical demonstration, and it deals with what we define as "urban diversity," specifically.

In the second section, "Broadening the framework," we proceed by broadening our initial approach, and we scale out.

In the third section, "Toward a unified framework of diversity mapping," we reveal interdisciplinary connections in our framework and discuss how scientists in any discipline can use the framework to pool their tools and methods.

Overall, the article is intended to be easy reading such that students at bachelor's and master's levels can read the article and develop diversity maps on their own. Since the article has been written, we have given it to students on multiple occasions, and they have succeeded in creating diversity maps (Della Pietra 2021; 
Raszka 2022). Part of the original article itself emerged in an educational setting as collaboration between an assistant professor and a student (Birchall 2021).

\section{Mapping urban diversity: rationale and example}

Diversity is a defining characteristic of cities. Early on, the founding figures of social science recognized diversity as something typical for cities (Simmel 1903; Maunier 1910). For example, the German scholar Georg Simmel observed that people who lived in large cities were exposed to a high diversity of stimuli. According to his theory, this diversity was one of the main sources of the magic spell of cities, and it led to the formation of a unique type of personality that he and his followers often observed primarily in city dwellers. Certainly, Simmel's work illustrates that diversity was key to theorizing about cities from early on.

Given this early recognition of diversity as a defining characteristic of cities, one would expect that the study of diversity is truly advanced in urbanism. However, although many urban planners have found interest in diversity, few have actually attempted to define and quantify it, and many have avoided studying it all together.

Some planners have posited that the mental image that people have of their cities is determined less by urban diversity than it is by great infrastructures such as towers or boulevards (Lynch 1960). This is certainly true in the sense that people can distinguish cities by looking for unique buildings and infrastructures: Paris has the Eiffel Tower, or Bilbao has its museum designed by Frank Gehry. If you see the Eiffel Tower in a movie, you think the scene is set in Paris. If you see a building by Gehry, you might guess that the city is Bilbao-although you might be wrong. These large infrastructures are certainly very important in making each city recognizable. Nevertheless, they might change over time. New York's World Trade Center was replaced by the One World Trade Center. The infrastructure has changed, yet the city and its diversity have remained. In a recent article, we have studied tall buildings in New York City, in particular, and we have shown that they can contribute to making the city diverse. There can be positive feedback loops in which vertical growth supports diversification and vice versa. In this case, too, the buildings are gradually replaced, while diversity stays or increases (Raszka and Baciu 2022).

Cities stay diverse even as large infrastructures are changing. This "golden rule of diversity" can be observed in a broad range of examples, some of which date back to antiquity. Legend tells us that people who experienced the city of Babel were stunned by two things: the Tower of Babel and language diversity. Certainly, the tower was great, but they also said that the actual name, "Babel," came from "babbling," which symbolized the presence of a great diversity of languages. What makes our point about diversity is that according to the same legend, the tower was abandoned, but language diversity has stayed. Diversity was also typical for medieval cities. Certainly, medieval cities had large infrastructures such as fortifications which were put in place for purposes of defense, but the richness of guilds and businesses that characterized medieval cities has stayed even 
though the fortifications have been removed as, for example, in Vienna, Zurich, and many other European cities (Mintzker 2012). Contemporary cities are no less characterized by diversity. Certainly, contemporary cities have large infrastructures such as traffic arteries and train stations, but cultural diversity stays even when the traffic arteries are turned into pedestrian promenades or bike paths as, for example, in New York City (Friss 2019). These notable historical examples are mentioned here because they demonstrate that the city is more than a sum of individual buildings or infrastructures. The city is diversity.

The golden rule of diversity, according to which diversity stays with the city, holds beyond the examples just mentioned. Almost every city is a center of urban diversity. Many nationally and cross-nationally established definitions of "city" are primarily focused on population density and diversity (Dijsktra and Poelman 2012). If something has urban diversity, you call it a city.

Although diversity is a defining characteristic of cities, one cannot always easily find and quantify it. If you take a moment to look at a satellite image of Europe taken by night, you immediately recognize the Blue Banana, a dense urban area that stretches from London in a slightly bent line (hence banana) through the Netherlands and along the Rhine to Switzerland and Italy. This area lights up quite brightly on the night images because it is very densely urbanized. However, the area is not only densely urbanized but also multicultural and multilingual, uniting six national languages and many different cultures. The satellite images do not reveal this linguistic and cultural diversity, although it may be a key factor that makes the Blue Banana one of the most active European centers of urban life (Baciu and Cellucci 2022).

With this observation in mind, it seems relevant to develop methods to quantify and map urban diversity. Before our work, nobody had actually indexed and mapped urban or cultural diversity. Thus, our work should be considered a primer in indexing and mapping urban and cultural diversity. We do not claim that our method is perfect, nor is it intended to be. There are no perfect methods; there are only methods suited for certain applications. Our primary goal is to provide a method that can serve researchers and students as a starting point in their studies, and we believe that this method is so easy to apply that everyone (including investors and city officials) could start creating their own diversity maps.

Let us begin by considering a concrete example — an urban area known as "Delfshaven" in the metropolitan area of Rotterdam. Delfshaven is a great place for us to study because it is situated in the upper top of the Blue Banana as well as near our research facilities in the Netherlands. This makes Delfshaven an ideally suited location for our study of urban diversity. Delfshaven also is an area of Rotterdam that offers a rich history that will help us validate our mapping. Most notably, Delfshaven is the place where the Mayflower Pilgrims, a group of fervent religious dissenters, left on the Speedwell to begin their voyage to the New World in 1620 (Sawyer 1925; Dupertuis Bangs 2020). They were among the earliest colonial settlers, and their journey has become a cultural symbol in the United States. Such a rich history can only be beneficial in this present study of diversity.

We will use Delfshaven as an example based on which we demonstrate how to index and map urban diversity. Although Delfshaven seems a good location to 
choose, the choice could have fallen on almost any other urban area. In subsequent articles, we also create diversity maps of several other urban areas such as Amsterdam (Baciu 2022), Athens (Nazou 2022), Copenhagen (Baciu 2022), New York (Raszka and Baciu 2022), or the UNESCO World Heritage Site of Sassi di Matera, Italy (Baciu and Della Pietra 2021a).

The next sub-sections propose a basic method for the evaluation and mapping of urban diversity. The method consists of six steps. Going through these six steps, we quantify urban diversity in Delfshaven, and, through historical evaluation, we show that the results are empirically meaningful. We begin with this basic method to make our article more accessible to the readers. The two subsequent sections ("Broadening the framework" and "Toward a unified framework of diversity mapping") will broaden this first, basic method, turning it into a general framework for the evaluation and mapping of diversity.

\section{Step 1, defining "urban diversity"}

Cities are centers of diversity. They are places where different parties interact in different ways. Based on this consideration, "urban diversity" can be defined as follows: Urban diversity is the diversity of different things that people do in the city.

Let us consider a few real-world examples of things that people do in a city. At first, these examples may seem mundane, but they illustrate what people do in their everyday lives in cities: they go shopping; buy their groceries; meet friends at a bar; go to a restaurant; go to a sports venue, a beauty salon, a cultural event, or a congregation; or they return home, etc. The list of such things that people do in cities remains open-ended. We would like to consider as diverse a range of activities as possible.

With these examples of urban activities in mind, our chosen definition of diversity may make sense to many people. Nevertheless, we would like to emphasize that this definition is only one of many possibilities. One could instead look for diversity in the morphology of the built structures, or in the materials used to build buildings. In principle, the choice of how to define urban diversity is arbitrary. The hope is that one can make a choice that later proves useful in one or another way.

\section{Step 2, collecting data}

Any empirical analysis of diversity must be based on empirical data. Given that our definition of urban diversity involves a broad range of urban activities, our next step is to look for data that record such urban activities.

Recording urban activities could require the creation of a complete record of every activity that takes place. For instance, every step that a pedestrian takes on their way through a city could be considered part of an urban activity that would have to be recorded in full detail. However, in our case, such a complete record is not necessary. Estimates of diversity do not need to be based on complete records of everything that takes place in urban space; they can be based on information that is most readily available. 
For example, activities such as "meeting friends at a bar" or "going to a beauty salon" require a bar or a beauty salon in the first place. This means that instead of recording all activities and evaluating them, we can simply look for the infrastructures where these activities typically take place. We can go on to count bars, beauty salons, etc.

The solution of counting bars, beauty salons, and the like is not perfect because the mere existence of a bar does not guarantee that it is used. Some bars may be very popular, while others stay empty. The popular bars host many urban interactions, while the unpopular ones do not. Of course, the popularity of a bar could be deduced from its revenue, and we could take such information into account. However, such a procedure would add another layer of complication, and we have proposed in the introduction that we would develop an easy and transparent approach for the mapping of diversity. Therefore, we stay with counting bars, restaurants, and other infrastructures, which is uncomplicated-and it may turn out to be sufficient for the present task.

Following these considerations, our first step is to look for different types of urban infrastructures. A record of these is easy to find. Consider Google Maps. It provides a quite detailed overview of bars, beauty salons, and the like. Countless people have contributed meaningful information to this platform, and many more use it to navigate the city. These people feel that Google Maps provides a good representation of their city. Such representation could be good enough for our first attempt to map diversity, too.

On close inspection of the Google Maps available for Delfshaven, we found that some businesses or infrastructures were missing. We therefore chose to improve this record by also taking a city walk through Google Street View, which allowed us to record any additional business or infrastructure that we found. Figure 1 illustrates how we have collected the data (Fig. 1).

\section{Step 3, performing the analysis}

Quantifying diversity requires an analysis of the data that are collected. Three main choices must be made for this analysis. One must choose:

(1) a classification system according to which the data are analyzed,

(2) a granularity at which the analysis is performed, and,

(3) a diversity index that quantifies the amount of diversity present at any given point in space.

Let us explain these three choices one by one.

\section{Classification system}

Researchers who wish to map diversity need a classification system to decide which activities belong to the same type of activity. In our case, we consider a broad range 

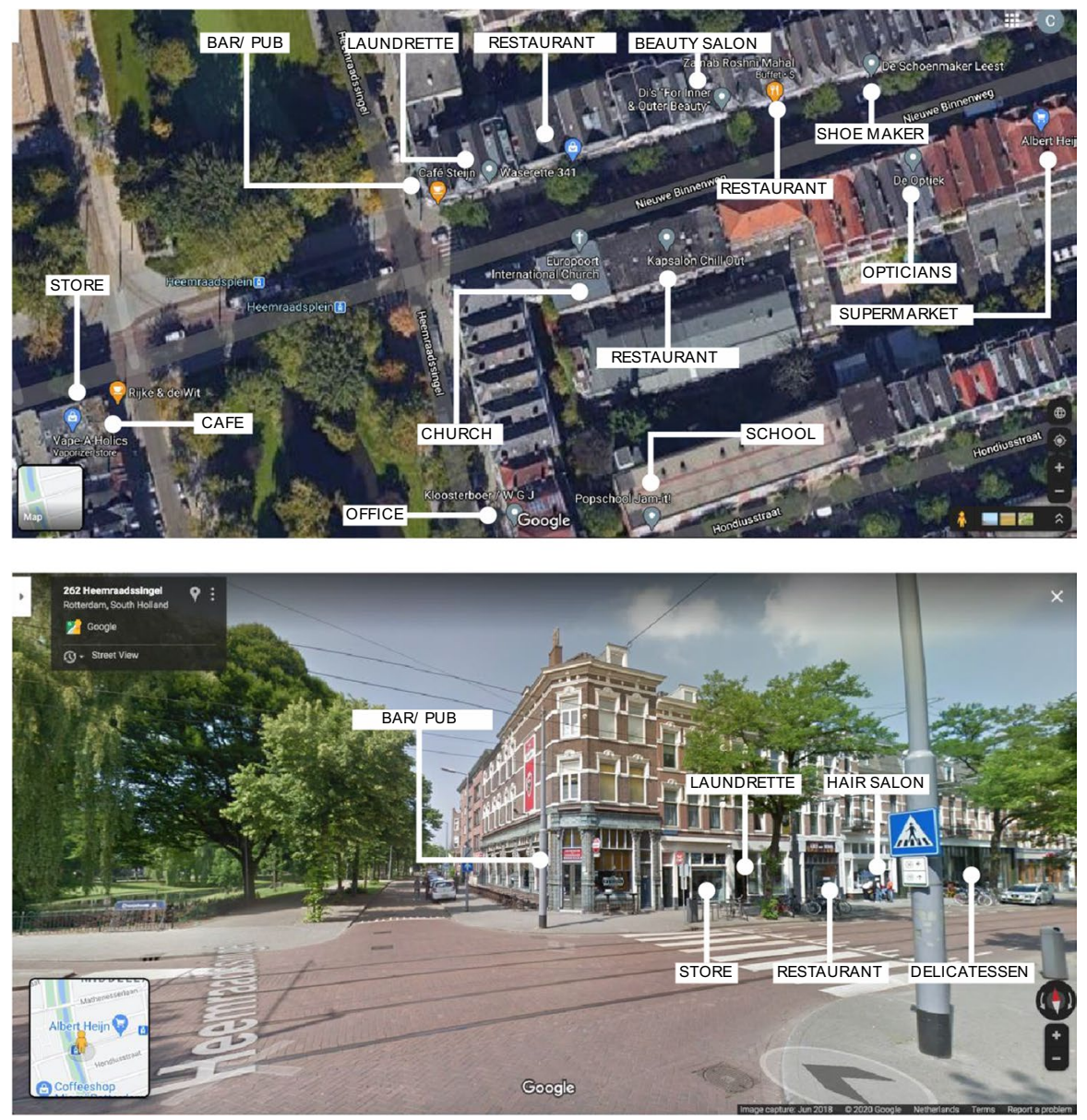

Fig. 1 Collecting data from Google Maps and Google Street View. We are looking for venues that house urban activities such as "going to a bar." Apartments and other types of dwellings are not marked in this figure, but they are considered as one type of activity in the analysis

of urban activities. We already mentioned activities such as meeting friends at a bar or going to a restaurant. What makes the classification necessary is that these two activities could be considered to be one joint type, given that they are very similar, or they could be considered to be two distinct types. The experimenter must determine which activities are considered similar enough to fall into the same categoryor distinct enough to fall into separate categories.

In principle, the choice of a classification system is arbitrary. Our choice is the simplest system imaginable; it is an architecture student's first intuition of what are clearly distinct types of activity. An overview of the resulting classification system is given in the section "Replicate the results".

Our classification system has a dozen distinct categories or "classes", but it could have hundreds. Technically, the total number of classes should be neither too high 
nor too low. It affects the results of the subsequent diversity mapping analysis in the following manner: in the extreme case in which there is only one class (all activities are of the same type), there is no diversity to consider. In contrast, in the other extreme case in which there are too many classes (each single activity represents a separate type of activity), the diversity map becomes a density map.

Classification systems can be developed based on the experimenter's knowledge, but they can also be developed algorithmically. Using a computer, one can perform dimensionality reduction on the data, which identifies natural clusters that can be used as classes in the analysis. This approach may be particularly promising when evaluating cultural diversity in urban space as will be discussed later in this article.

The value of a classification system is often known only in retrospect, after it is applied in research. A good system is one that provides useful analyses.

\section{Granularity}

The next important choice is the granularity at which the analysis is performed. Given that we have chosen to evaluate activities such as "going to a bar," the granularity of the analysis is as good as defined, namely as the building. Although this choice seems obvious, it actually is arbitrary, too. One could just as well go into more detail and look at every single square meter of a city, or one could zoom out and consider only entire streets. Here again, good choices lead to useful analyses.

Choosing granularities also involves deciding whether each item that is analyzed (in our case each building) must necessarily fall into only one single category, or whether it can fall into multiple distinct categories. For example, a building could be classified as "bar." But it could also be classified as "partly bar, partly shop, and partly housing."

For the sake of simplicity, we shall choose to represent only one type of activity for each building. If one building houses multiple venues that allow for different activities, we shall select the most rare type of activity as the one that defines the building. Of course, this choice is also a mere convention and may vary from experiment to experiment.

\section{Diversity index and scale of the analysis}

Finally, it is important to choose a diversity index. The diversity index is used to assign a diversity score to each point in urban space. In our present example of Delfshaven, let us proceed with the simplest possible approach. We say "simple" because this approach is very easy to perform, and it can be easily verified through human inspection.

We overlay the urban map of Delfshaven with a fine-meshed grid, which represents the scale of the analysis, and we define our diversity index as the number of different types of urban activity found in each field of the grid (Fig. 2). Technically, this approach is equivalent to measuring "richness" in the life sciences.

A more complex diversity metric that we could employ is Simpson's diversity index. Applying Simpson's index would provide the advantage of reducing scale 

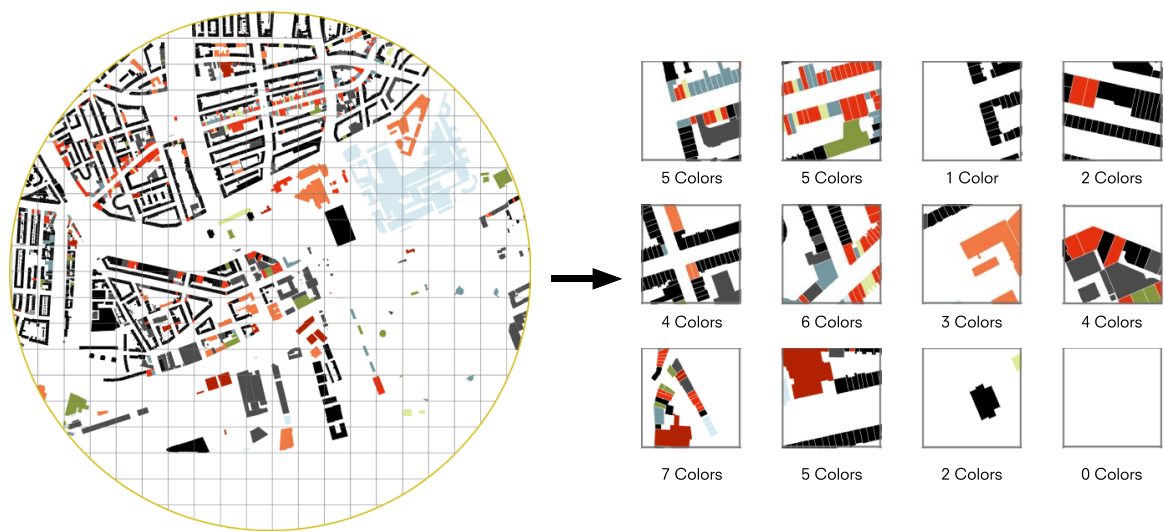

3 Colors

4 Colors
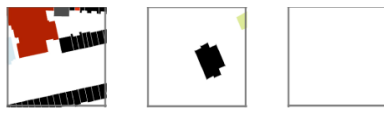

5 Colors

2 Colors

0 Colors

Fig. 2 The collected, processed, and mapped data, overlaid with the analysis grid. The diversity index employed here consists of counting how many different types of urban activity appear in each field (size $100 \times 100 \mathrm{~m}$ ). The size of each field is taken to represent the amount of urban space that a person perceives around him/herself at any given point in time

dependency. For example, if, in Fig. 2, we decided to make our grid more broadmeshed $(200 \times 200 \mathrm{~m}$ instead of $100 \times 100 \mathrm{~m})$, each field would contain more types of urban activity. We would find more diversity in the same urban space, although the data under consideration are the same. This effect can be diminished by choosing an index that is less scale-dependent. However, the cost of using such an index is that it makes the approach less straightforward. We will use Simpson's index in later examples in Figs. 7 and 10, and we also use it in other articles (Baciu and Della Pietra 2021b, Raszka and Baciu 2022).

\section{Step 4, synthesizing the results obtained from analysis}

At first, the results obtained from diversity analysis are just numbers. A complete evaluation of diversity must necessarily involve ways to represent and make sense of these numbers. For example, we can create a diversity map, which makes the numbers intuitive to interpret.

In principle, there are many other ways to synthesize and visualize the output obtained from the analysis. One can print out the data in full text, or one can look for minima or maxima. If you ask us for a good way to get started, we suggest starting with maps because they are intuitive. Researchers have shown that the human brain has a special sense of geography, and maps are very frequently used in both architecture and urbanism. Architects and urban geographers know how to interpret maps. Let us therefore go ahead and map the results. We will produce a map layer that indicates diversity. High diversity is indicated by dark red, low diversity by bright yellow (Fig. 3).

Now that the results are neatly represented on a map, let us in more detail explain our motivation for creating such a map. Here is some context: many maps use colors to indicate different types of information. For example, the hierarchy of different types of road (from highways to byways) is often coded in colors (highways 

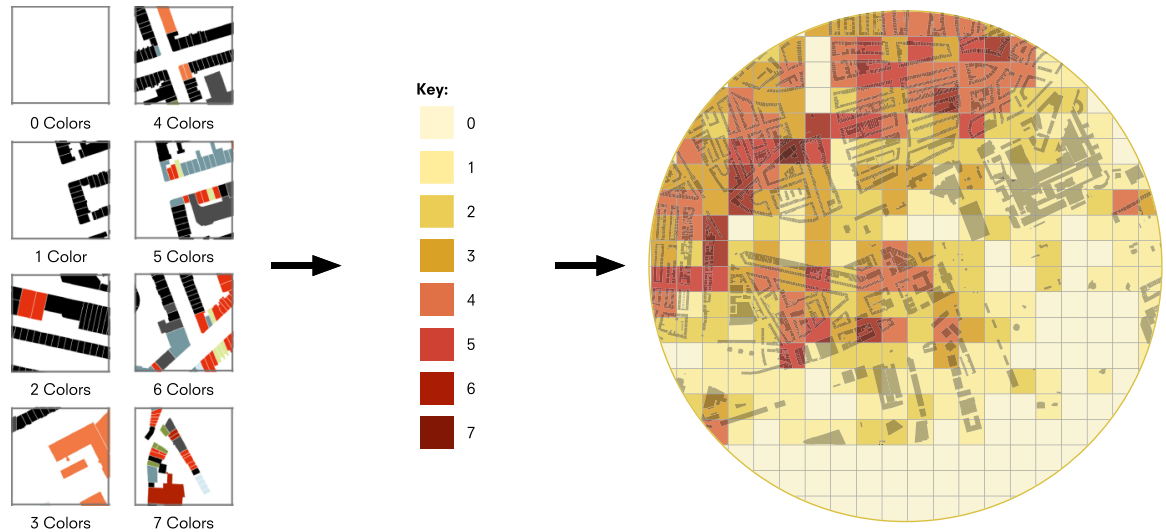

Fig. 3 Creating a diversity map from the results obtained in the analysis. The map may be the most intuitive tool for architects, yet the processed data can also be used in other ways, such as for additional computation and prediction

are red, large roads yellow, small roads white). This color-coding makes it easy for any architect or urban planner to interpret a map. Architects and planners know that large roads are loud, often inviting more aggressive and fast driving than small roads. Such information is valuable; it tells something about the qualities of a given location on the map.

In our case, we use color-coding to indicate diversity. The new map layer that we create says something about the range of different urban activities present in a given urban context. Such information may be relevant; almost all built environments have buildings, but not all built environments are diverse urban centers. Our map locates diversity and, through this approach, it may help architects find urban centers. Architects may want to let the information that they gain flow into their designs the same way they consider information about street sizes, noise, and speed limits.

The diversity layer that we have created may be particularly useful because it cannot typically be decoded from urban morphology alone. Here is what we mean: highways have characteristic shapes. On maps, one can recognize most highways as long, gently curved lines. The same cannot be said about diverse roads compared to those that lack diversity. Typically, an architect requires several city visits to understand how diverse an area is. Our diversity map could help architects obtain such information prior to ever visiting a city. Of course, that's all theory. To understand whether our map can be such a support, we must next validate it.

\section{Step 5, validation: are the final results a good estimate of diversity?}

Before we can go ahead and use the maps, it is advisable to first validate them. In theory, we have succeeded in mapping diversity, but we don't know whether we have also succeeded in practice. Thus, we had better find a way to double-check our success. 
In order to go ahead with double-checking, we must first take a step back. We must return to the definition of urban diversity given in the section "Defining urban diversity", and, based on this initial definition, we must find another, independent way to locate urban diversity. We can then compare the results obtained through the initial evaluation and through the path chosen for double-checking. Here is how we proceed:

Logically speaking, urban diversity, as defined in the section "Defining urban diversity", has most time to emerge in urban centers that have long been active. Activity means interactions, and, in the long run, interactions make most sense between different parties (Baciu 2015). This leaves us with the question of whether the areas that we identified as centers of diversity also are among the urban centers that have historically been most active. Can we locate such urban centers through a method that we did not use when mapping diversity?

In the case of Delfshaven, the location of the historically most active urban centers can be found through historical evaluation. Evidently, historical evaluation is a method ideally suited for the task of locating historical centers, and it also is independent of our mapping method; it uses historical data rather than present-day maps. Historical evaluation is therefore a good fit in our attempt to validate our maps.

Historical validation is straightforward in the case of Delfshaven. The fact is that Delfshaven remained intact after World War II. Thus, we expect that urban centers have formed slowly and that they have mostly stayed in place.

Let us briefly review the history of Delfshaven, since it has been urbanized. Delfshaven is located at the confluence of the Nieuwe Maas and the Schie. The Nieuwe Maas is the main river that flows through the center of Rotterdam, while the Schie was initially a swampy creek that was dug out when the city of Delft was built. This made the old center of Delfshaven (literally "Delft's haven") an important location for trade. Later, the Schie was connected to the Rhine via the Rhine-Schie Canal, which allows ships to go all the way to Leiden. Delfshaven thus became a node in a nautical network between Rotterdam, Delft, and Leiden.

The old center of Delfshaven is probably most famous for the Oude Kerk, literally "old church," also known as Pelgrimvaderskerk or "Pilgrim Fathers Church." The Oude Kerk is important in our story because it is the place where the Mayflower Pilgrims embarked upon their voyage to the New World.

Of course, the sailing of the Mayflower Pilgrims was an exceptional event in Delfshaven's history. Most people did not sail to the New World, although they were involved in port activities, and they mostly traveled to Rotterdam by land. This detail directs our attention to the road between the Oude Kerk and the center of Rotterdam. This road is the place where active urban life formed first and had most time to diversify. This observation can be further strengthened though evaluation of historical photographs. On historical images, we see large crowds gathering in front of theaters, or we see people walking and window-shopping.

Our idea is to now look up this road on our diversity map. We would expect that this active urban center has historically been and has remained a diverse urban center. Does it rank high on our map of urban diversity? The answer is, "Yes!" 

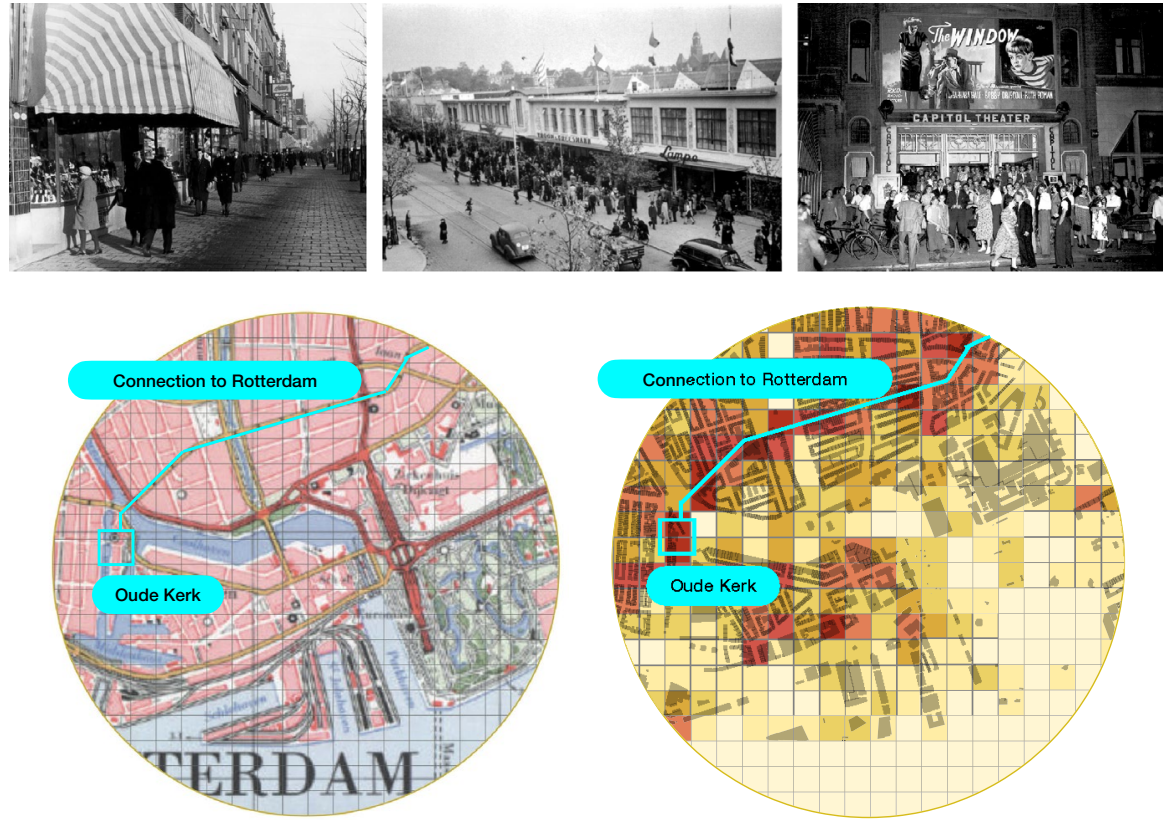

Fig. 4 Through historical evaluation, we identify the urban area on our map that has been most active over the last centuries and where diversity has had much time to form (marked bright blue). When locating this area on our diversity map, we find a maximum in present-day diversity, as expected. Thus, the historical evaluation validates the mapping results. Above Historical photos taken in 1936 (left), 1940 (center), and 1951 (right). The photos document high urban activity, both day and night

urban diversity reaches a peak in exactly this area. Thus, the historical evaluation validates our diversity mapping (Fig. 4).

There is a second and equally important way to validate our mapping results, namely by looking at streets that lack urban diversity. Let us now identify an area that historically lacked diversity.

The Hoboken area is a large plot of land that has historically been cut away from urban life; it was long owned by people who kept it private (Fig. 5 above). Urban life in this area started to form only recently, and urban diversity is therefore expected to be low. This latter proposition is at once confirmed when the Hoboken area is located on our diversity map. The Hoboken area ranks lowest in diversity (Fig. 5). Thus, historical evaluation validates both maxima and minima among our mapping results.

\section{Step 6, employing the empirical results}

Now that we have validated the mapping results, the question is what one can do with them. Certainly, the results have little value, unless they become useful in practice in architecture or urbanism, or toward the development of theory. 


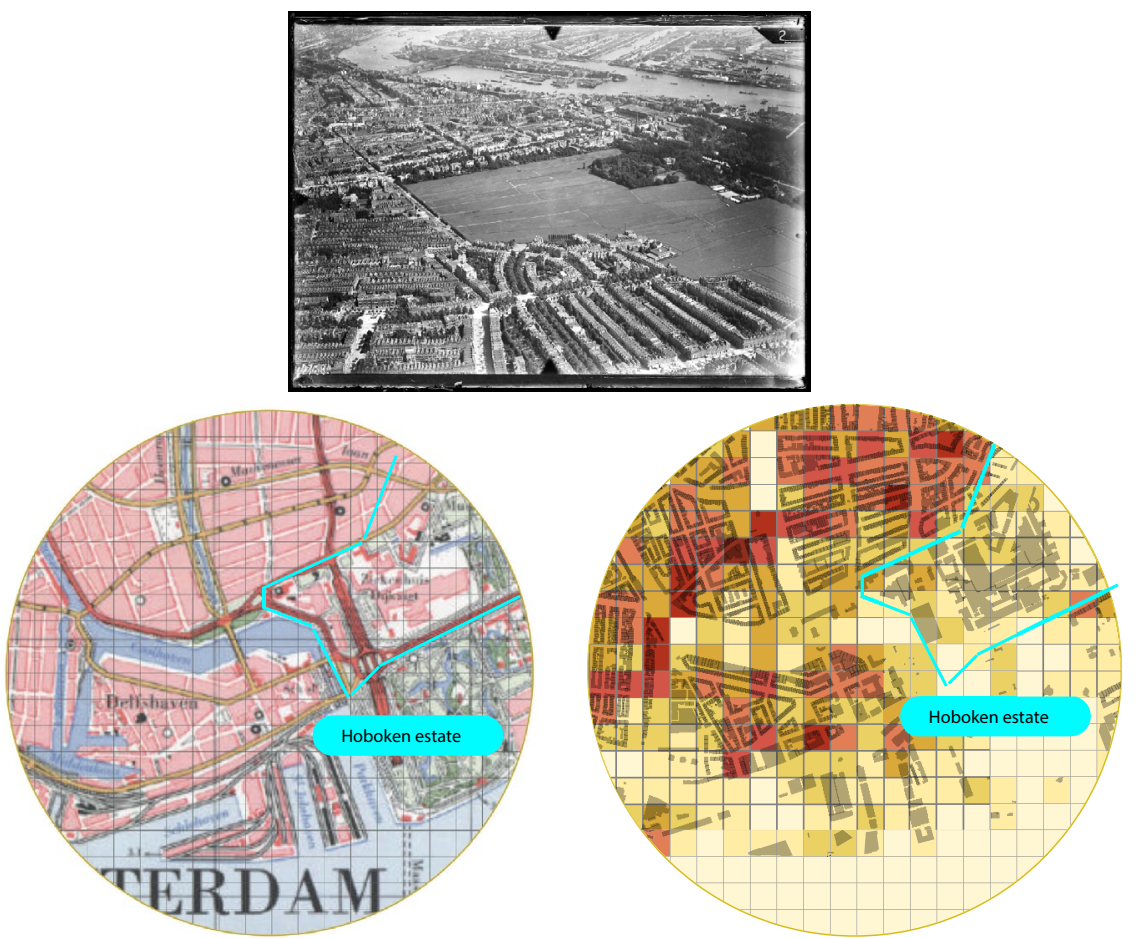

Fig. 5 Historical evaluation suggests that the area marked blue has had little time to diversify. Our diversity mapping indicates that this area ranks lowest in diversity today. Here again, the historical evaluation validates the diversity mapping results. Above Aerial image of Hoboken estate around 1930, illustrating the historic lack of urban life. The area was private and has only recently been opened to public activities

Practical applications are the ultimate goal of much science. In "Step 4", we have already considered how our diversity map could be useful for architects and planners. We would now like to continue those considerations and demonstrate how our diversity mapping method can be used as a design tool.

Diversity mapping helps architects not only locate some self-defined type of diversity but also determine which of their project variants most increase the diversity that they have located. All that is needed is to insert the project variants into the map, re-evaluate diversity with each variant, and compare the new results (Fig. 6).

In research that we have performed since this article was first made available as an online preprint (Baciu et al. 2021), we have demonstrated that such an evaluation can also be performed to help planners or developers locate ideal places to intervene. For example, a developer who is planning to build a hotel may want to know where the hotel could most increase urban diversity. Such an interest could be economically motivated: if a hotel increases urban diversity, it may support an increase in land value, which could return financial benefits on the investment. Our contribution in this subsequent research was to demonstrate how to locate areas where a particular type of building increases or reduces diversity at a given point 

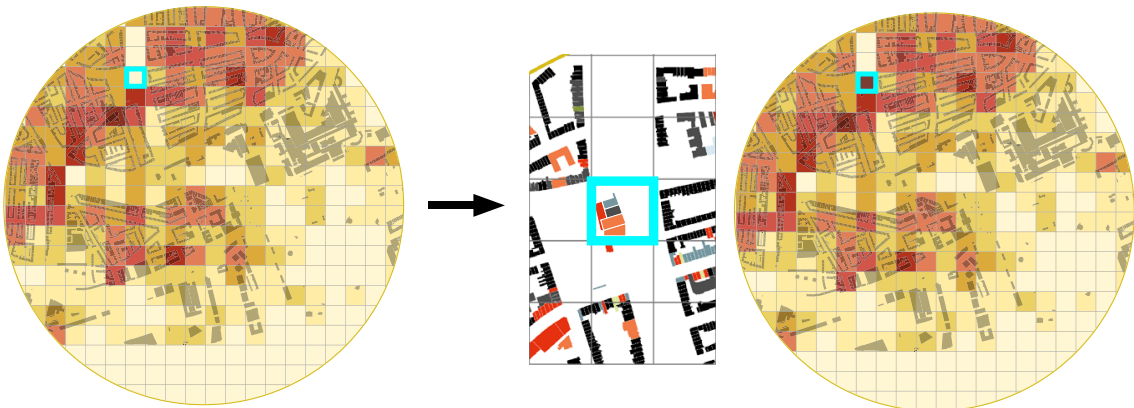

Fig. 6 Through diversity mapping, architects can evaluate the effect of a proposed project. Left diversity map used to locate a given type of diversity. Right proposing a project to enhance the diversity that was found. (The new evaluation result is obtained by performing the diversity mapping once again after a newly proposed project is inserted into the original data)

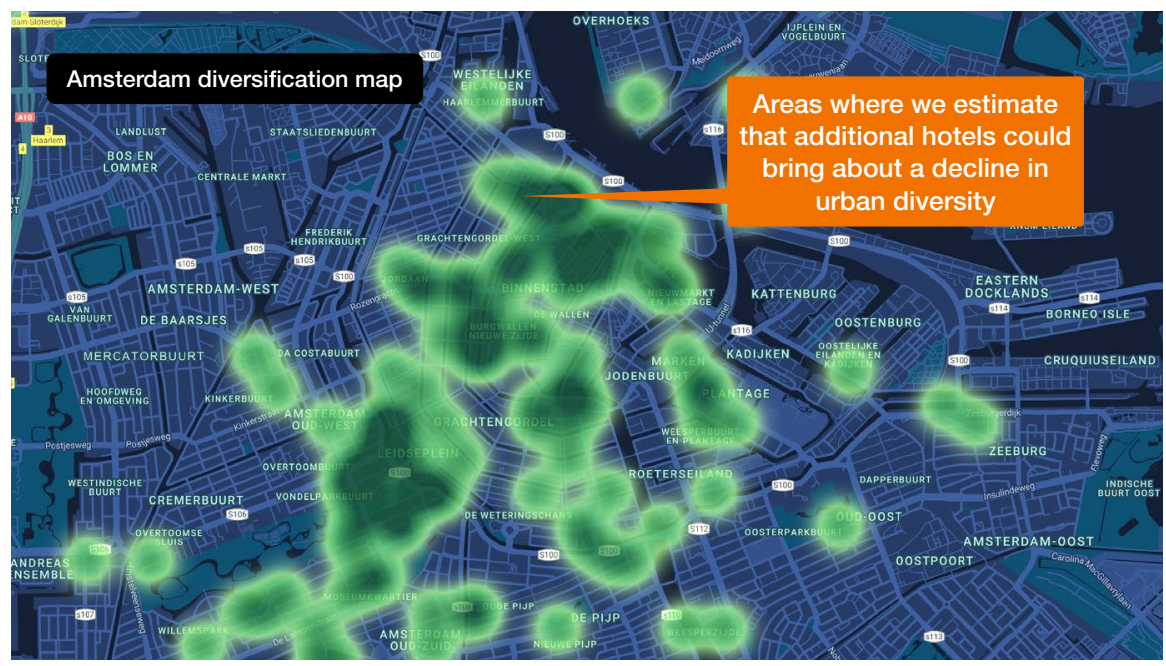

Fig. 7 In our subsequent research, we performed diversity mapping and we simulated how diversity may change over time in Amsterdam. The dark green areas in the figure are places where additional hotels would bring about a decrease in urban diversity if built in 2022. We expect that developers may gain the most profit from building hotels outside these dark green areas, considering that a diverse city may be more profitable than a "touristic city" in which tourism predominates (Baciu 2022, see also Loevezijn 2022)

in time. Figure 7 shows such a map, taking Amsterdam as a case study. Amsterdam is on the verge of becoming a touristic city. Figure 7 shows where urban diversity decreases if new hotels are built (Fig. 7). We call this type of map a "diversification map"; it indicates how diversity is expected to change in response to other changing circumstances. 
Next to practical applications such as the ones just demonstrated, our mapping method can also be used for the development of theory and even for the advancement of science at large. To explain why our maps are useful toward this end, we would now like to describe a case in which diversity maps like ours can be used toward the development of theory and toward mathematical descriptions that apply across multiple sciences.

As we have already observed, urban diversity may sometimes form very slowly. Even so, the amount of diversity that is detected in a given point in urban space may change over time. We call this process "diversification," and we have illustrated it in Fig. 7.

Diversification can take positive or negative values. "Positive diversification" takes place when diversity increases. A peer reviewer who read our article imagined this process as a maturation process of the city. The city becomes at once more diverse, more attractive, and more resilient to change. Positive diversification is valuable. However, diversity may also decrease, in which case we speak of "negative diversification."

We are not the first researchers to observe that diversification takes place in urban environments. In 1961, urban activist Jane Jacobs became famous when she published her book The Death and Life of Great American Cities (Jacobs 1961). In this book, she often discussed changes in diversity. She described how diverse urban street life attracts economic growth, yet growth has a counterintuitive effect. It diminishes diversity. Jane Jacobs's work inspired a flurry of subsequent activism and research. Clearly, the loss of diversity that she observed is a case of negative diversification.

The big question now is whether the causes for such positive or negative diversification can be exposed, and whether the connection between causes and subsequent diversification can be described with mathematical formulas. Based on our previous work, we believe that such causal modeling is possible.

In previous work, we studied diversification in a different context. We studied cultural diversification, and we demonstrated that it can be modeled with standard life science equations (Baciu 2020a, b, 2019, 2018). We are now proposing that urban diversification can be modeled with the same equations.

In the initial preprint of this article, we did not offer data to test this proposition. Our only aim was to name an example that could document that diversity maps can be useful in advancing science and in helping researchers make interdisciplinary connections. Our point was clear: multiple diversity maps are necessary to study urban diversification simply because such research must cover not just one moment in time but an extended period of time. In turn, studying urban diversification could help researchers make connections to diversification in other fields of study.

Since this article first appeared in preprint, we have moved ahead and analyzed urban diversification in the UNESCO World Heritage Site of Sassi di Matera (Baciu and Della Pietra 2021a). Sassi is a picturesque historical settlement of astonishing diversity and beauty. Sassi's history stretches over several millennia, but in the 1950s, it went through a dark and dramatic age when urban planners who did not sufficiently understand or value diversity evacuated the settlement. For the planners, Sassi was a ghetto. They believed the settlement was too messy or simply too 
diverse. Through the enactment of a law that was rather strictly enforced, the inhabitants were forced out. The ensuing history proved the planners wrong. Sassi has meanwhile come back to life as a picturesque and diverse urban center.

We studied diversification in Sassi since it was resettled. We observed that together, the new settlers and the tourism industry brought an increasing amount of urban diversity, which made the settlement increasingly attractive; it was eventually listed as UNESCO World Heritage Site in 1993. In addition, Matera, the city where Sassi is located, became the European Cultural Capital of 2019.

At this important point in history, two opposed but enlightening events took place. On the one hand, officials believed that tourism was beneficial for the city, and they supported additional growth in the tourism industry. Their belief was based on experience from previous years in which they saw that tourism had constantly been beneficial. They did not believe this constancy could change. Locals disagreed. They went on to individually protect their buildings, which effectively blocked them from becoming hotels. The question is, who was right: the officials or the inhabitants?

Our diversity mapping and simulation suggests that the inhabitants were right. When we simulate a further increase in the number of hotels, the consequence is no longer an increase in urban diversity. Instead, diversity decreases, which is what the inhabitants avoided through their wise actions. With their intuitions, the inhabitants chose "city" over "touristic city" believing the first to be more valuable.

Jane Jacobs would have likely been interested in our results. As already mentioned, she observed that urban diversity attracts growth, but growth reduces diversity. The reason is that growth is often uneven in different industries, which results in an unevenness that undermines urban diversity. The most successful industry comes to predominate. Jacobs wrote:

"The duplication of the most profitable use is undermining the base of its own attraction, as disproportionate duplication and exaggeration of some single use always does in cities" (Jacobs, 1961, p 245).

In this and similar sentences, Jacobs suggests that diversity makes an urban environment attractive, and attraction brings growth to this environment. The problem is that, ironically, attraction is most profitable for those who are the most attractive within the environment, which leads to uneven growth that undermines diversity. The most attractive industries come to predominate, and they keep expanding until there is little else left. At this point, this initially attractive urban environment has lost its diversity, and with it, its initial attraction. (Thinking of our Italian site and of diversity as something that brings attraction, we are reminded that the Italian word for enjoying, "divertirsi," has the same etymological root as diversity.)

When we read such disillusioned writing, we begin to understand that urban activists long intuited that urban areas go through entire cycles of diversification. In such cycles, diversity attracts growth, but growth is uneven, and uneven growth reduces diversity until diversity is lost and the growth stops. According to our theory, diversity can return at this point, and the cycle can start all over again.

Sassi di Matera is a case in point where we can observe through empirical data and simulation how urban centers go through diversification cycles, and how multiple parties act together, sometimes trying to break the potentially negative impact of 
such cycles. Finding balance in a diversification cycle is not easy, but our diversity mapping method might help in future cases. The only rule of thumb that planners would have to follow is "keep the city diverse." An early-warning system could be put in place that could help communities find balance or even stability in diversification cycles that risk becoming too strong.

As already mentioned, such diversification cycles are not unique in urbanism. In our previous work, we have observed and quantified the same cycles in culture (Baciu 2019, Baciu 2020b, Baciu and Kahnt 2015). For example, scientific culture has gone through three diversification cycles over the last three centuries. During this time, science has gone through three periods of growth during which the diversity of scientific fields has decreased. As expected, each period of growth was followed by a period of reform, during which the diversity of scientific fields has rebounded to its initial values (Baciu 2020a).

We also found the same diversification cycles in the growth and maturation of institutions such as the University of California System (Baciu 2020a, b). The cycles are also found in virology (Nowak and May 2000) and in ecosystems (Holling's figure-8 model of ecosystems). The causal explanation and the mathematics for the cycles remain the same in all of these cases (Baciu 2021a, 2021c, 2021d).

While our initial preprint did not offer empirical data for diversification in urbanism, our subsequent work has performed this task and is shown here in Fig. 8. The point that we made in the initial preprint remains the same, namely that without diversity mapping, it would be difficult to study diversification cycles. And without diversity mapping, one would potentially miss out on a truly broad connection between very different sciences. The benefit of the connection is that it helps researchers pool their insights. Some aspects of diversification are best seen in one discipline while others are best seen in others. Pooling the insights from many distinct disciplines can help researchers develop better theories and give explanations that apply across a broader range of subjects. In this sense, we believe that finding one explanation that explains everything is more valuable than staying with many isolated explanations that explain only isolated aspects of isolated things. Compared to those isolated explanations, the broad explanation that explains everything is more likely to explain something entirely unknown and drive the development of new research and technologies.

\section{Broadening the framework}

Given that diversity is an index value, it is easy to see that multiple types of diversity can be studied separately or in unison. One can estimate urban diversity just as we did, or one can choose different definitions, different empirical data, different granularities, different classification systems, and different diversity indices, and one can easily merge multiple sets of results. Figure 9 shows results obtained from three different evaluations.

In this article's introduction, we mentioned that we would broaden our method, making it applicable to the study of cultural diversity. This can be done through the 
Fig. 8 Diversity maps for Sassi di Matera show how urban diversity has increased over the last few decades. The number of hotels has doubled several times over, which initially supported diversification, but a further doubling of the number of hotels would bring about a decay of urban diversity. Above Touristic presentation of Sassi as found online at Costa Crociere

evaluation of cultural data such as texts collected from news and books. The idea here is to create diversity maps that reflect what people think and say about urban spaces. One can collect a corpus that represents some type of culture, and one can automatically detect diverse groups of ideas and map them. We previously introduced this possibility in another article (Baciu 2020a, b). The step that we are now proposing as an addition to that previous research is to continue this analysis by computing diversity indices. Through this step, an urban map of cultural diversity can be created. The result is shown in the upper part of Fig. 10. An interactive visual can be accessed through the link provided in the image caption. The map covers the entire globe.

Similarly, it is possible to analyze diversity based on large collections of images. In the lower part of Fig. 10, we have created a map that quantifies the diversity of architectural styles across the United States. The map was generated based on the image collection of the Society of Architectural Historians. Since our present article has been made available as preprint, we have gone ahead and discussed the theoretical meaning of these maps in more detail in another publication (Baciu 2021c, d).

The maps shown in Figure 10 cover a larger geographical range than that covered in the Delfshaven example. Such large maps can also be created for urban diversity with the basic method developed in the section "Mapping urban diversity: rationale and example". The record that we used in that section (Google Maps and Google Street View) is available worldwide, and methodologically, the only requirement is to train a computer to recognize different types of venues: restaurants, bars, etc. This task can already be done with sufficient accuracy. Thus, producing a global map of urban diversity, and keeping it up-to-date, has now become primarily a matter of computational resources. Evidently, our initial method for diversity mapping can easily be broadened and scaled out for high-resolution diversity mapping with regional and global coverage.

\section{Toward a unified framework for diversity mapping}

We have already mentioned that researchers have studied diversity across many scientific disciplines and that some discoveries were made multiple times independently. One of the most notable examples of such discoveries is Simpson's diversity index. Simpson's index was initially formulated in ecology and linguistics (Fisher 1943; Yule 1944; Simpson 1949), yet it was independently formulated in economics (Hirschman 1945), and it later found applications in politics (there known as effective number of parties), in physics (there known as inverse participation ratio), and in virology (Nowak 1991). Furthermore, our own work in the humanities and in urbanism has theorized and empirically shown that Simpson's diversity indices can 

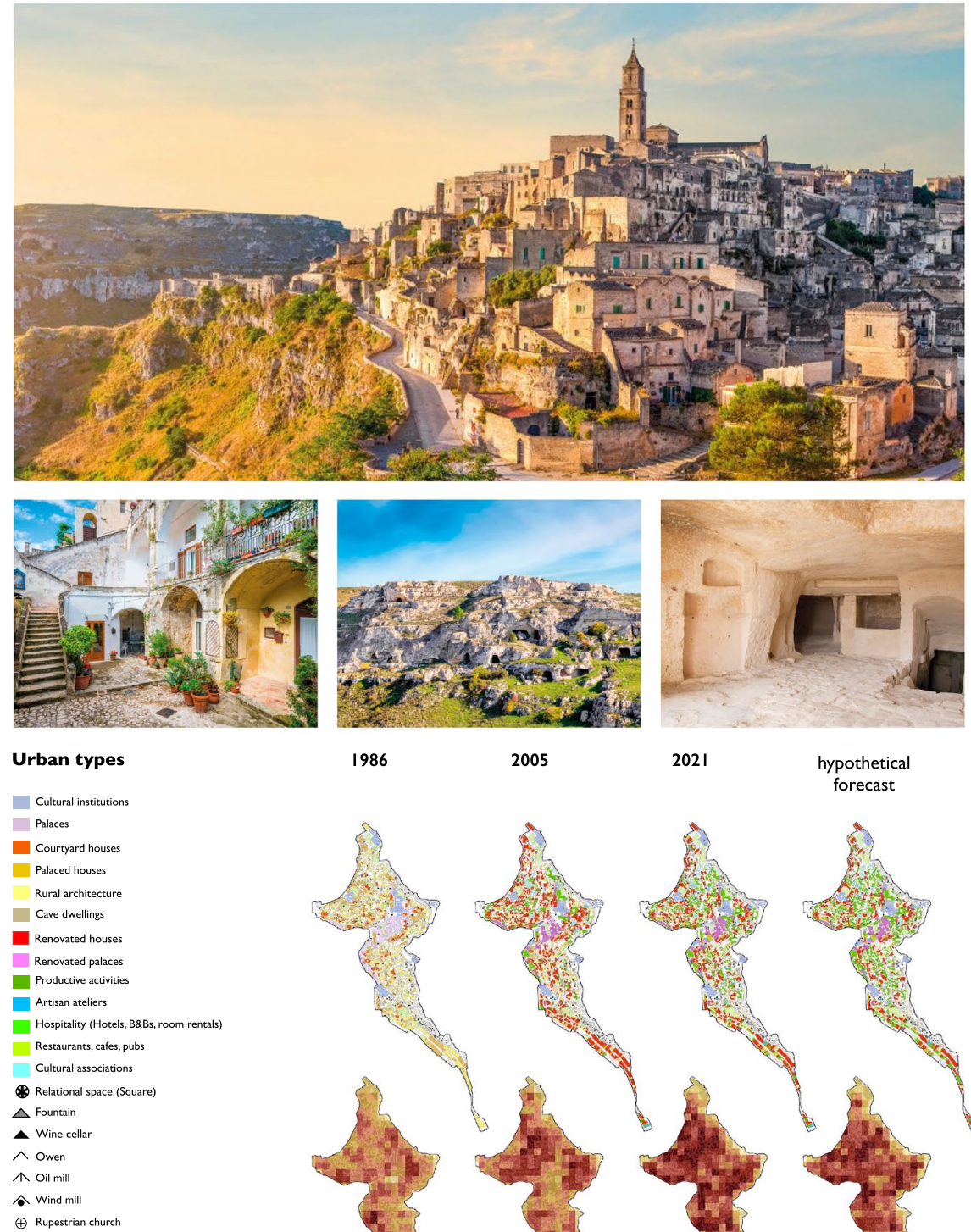

\section{Diversity Analysis}

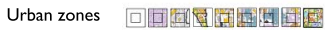
Nr. of types $\begin{array}{llllllllll}0 & 1 & 2 & 3 & 4 & 5 & 6 & 7 & 8\end{array}$ Diversity $|+| 1|1| 1 \mid$

1986

2005

2021

hypothetical forecast

Colour code
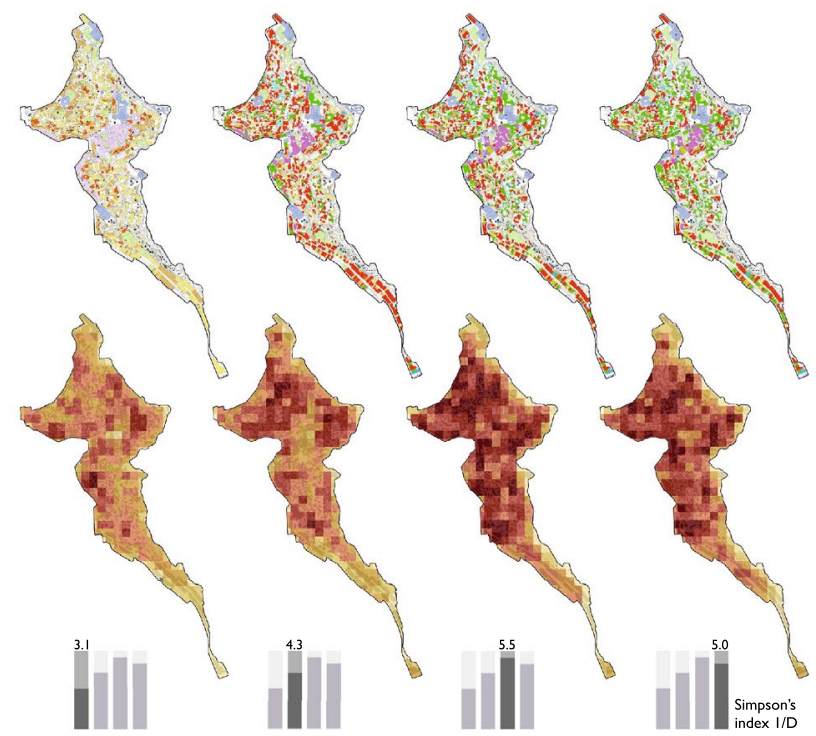

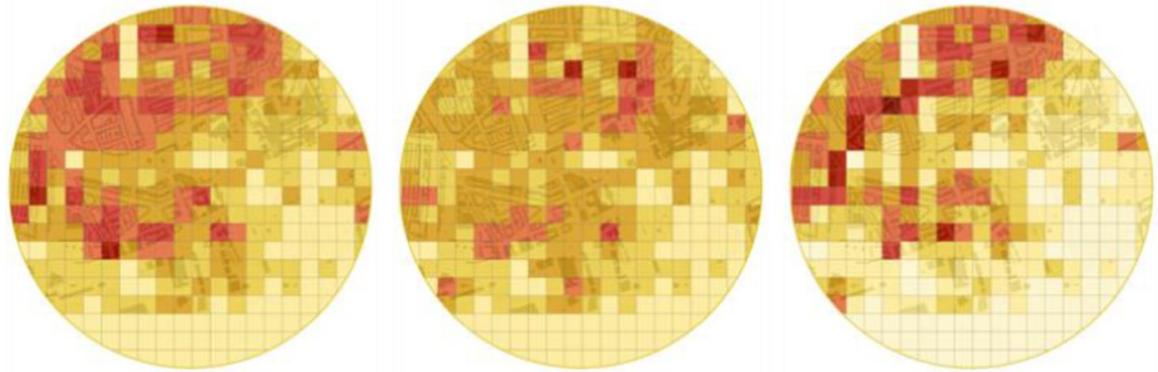

Fig. 9 Maps representing three different types of diversity. From left to right sizes of buildings, building materials, and urban diversity
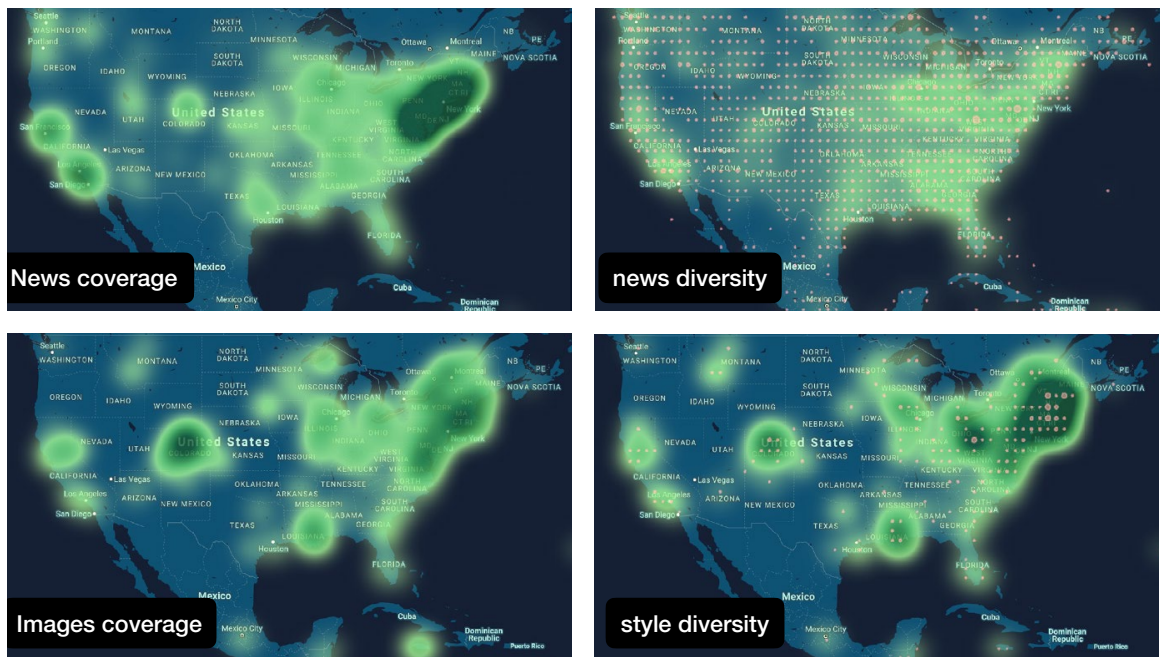

Fig. 10 Density and diversity maps for culture and architectural styles. Above maps representing the density and diversity of US news that mention the term "science." Left news density (how much coverage there is for each area). Right news diversity (in how many different news topics does science enter, computed as Simpson Diversity Index 1/D). As can be seen, New York, LA, and San Francisco are by far the most-covered urban centers, but science enters a similarly broad range of news topics in other urban centers, for example in Georgia or along the entirety of the West Coast. Interactive visual: https://mappingdiversity.herokuapp.com. Below maps representing density of historically relevant buildings and diversity of architectural styles based on data curated by the Society of Architectural Historians. Left density of geocoded photographs in the collection. Right diversity of architectural styles among the geocoded photographs. Interactive styles visual: https://diversity-styles.herokuapp.com

play an important role in predicting diversification cycles in human culture and in urban space (Baciu 2020a, b).

Since the first preprint of this article was published, we have pointed out that the success of Simpson's index has a broad and fascinating basis. To explain our insights, we must briefly introduce readers to the index. Simpson's index is a quantitative measure for diversity. Mathematically, Simpson's index represents the 
probability that two randomly chosen items belong to different categories (Baciu 2020a, b). As a concrete example, imagine picking colored marbles from a bag. If you always pick same-colored marbles, color diversity is low. In contrast, if your marbles are always different-colored, diversity is high.

Technically speaking, Simpson's index is calculated through pairwise multiplications (one multiplies all pairwise numbers of items that fall into any two different categories), and exactly these multiplications are the mathematical characteristic of almost all models used to express interplay, which is the root of diversification (Baciu 2020a, b, 2021a, 2021b). No matter in which field you study diversification, you will likely feature multiplication signs in your causal models. This counts for all compartmental models in fields such as epidemiology, virology, or humanities, as well as for the replicator equation of game theory, which is equivalent to generalized Lotka-Volterra equations. These latter equations are mentioned here because they find applications in almost all sciences. Thus, studying diversity and diversification in almost all sciences can be done with the same mathematical operation: diversity and diversification can be described with multiplications. This insight will certainly appear intuitive to readers if we remind them that multiplications are not only used to describe diversity and diversification, but also multidimensionality more broadly-and we intuitively imagine diversity as some sort of multidimensionality (Baciu 2020a, b, 2021d). Based on these considerations, we begin to understand that the study of diversity and diversification may unite all sciences.

The question that we already addressed in the original preprint of this article only gains in relevance. It can be summarized as follows: would it not be valuable if discoveries made across multiple sciences could be pooled together such that scientists could learn from each other? If so, how could such interdisciplinary pooling be achieved?

Let us now sketch out a unified framework for the evaluation of diversity. The idea is to create a framework that helps researchers exchange tools, techniques, and theories. The backbone of our framework is easy to understand; it consists of the six steps that we went through in the section "Mapping urban diversity: rationale and example." The steps have proved useful, which motivates us to keep them. When evaluating diversity, we suggest that it is mostly advisable to go through the following six steps:

(1) Define the type of diversity to be studied.

(2) Collect data.

(3) Perform the analysis by applying a system of classification (3.1) at a given granularity (3.2), and by computing a diversity index (3.3).

(4) Synthesize the results obtained from analysis, for example by mapping them.

(5) Validate the synthesized results through a path independent of the one used in the analysis.

(6) Use the results for a given application or for the development of theory. 
We believe that pooling methods can be useful on the grounds that pooling could help scientists profit from each other's findings. In each of the proposed steps, rich connections can be found that unite the life sciences with the humanities and with the sciences of the city. For example:

In step 1, we argued that diversity is a defining characteristic of cities, yet high diversity is also found in the centers of ecosystems. More broadly, diversity is a characteristic of almost all life in both human-made and natural environments. It would seem relevant that scientists discuss their main observations across disciplinary boundaries. Why is life always diverse (Baciu 2021a, b)?

In step 2, we have chosen Google Maps and Google Street View as a record of urban infrastructures and therefore also as a record of urban activities that they house, yet such maps and photographic records are also used in the study of ecological diversity (Gould 2000; Schweiger and Laliberté 2022). Diversity is often studied through remote sensing. Thus, techniques for remote sensing should be discussed across disciplinary boundaries.

In step 3.1, we proposed that the classification system to be employed in the analysis of diversity does not always need to be formulated through human inspection. Instead, classification systems can sometimes be obtained through automated analysis of natural clusters. The natural clusters are units of evolutionary selection and therefore relevant for the study of diversity (Baciu 2021a). This insight also applies to ecology: genotypes of different species can be distinguished from each other through automated analysis of natural clusters. These clusters coincide with biological units of evolutionary selection (Pritchard et al. 2000). It would seem relevant that scientists across disciplinary boundaries discuss the development of classification systems.

In step 3.2, we mention that it is relevant to choose a granularity at which the analysis is performed. This choice may strongly affect the diversity evaluation. For example, in the early 2000s, investors believed that it was important to diversify one's portfolio. Each investor eventually had a diverse portfolio. Yet a closer look at this development reveals a problem. If the granularity of analysis is the assets inside an investor's portfolio, there is high diversity. The problem was that all portfolios became the same diversified portfolio people thought to be ideal. Thus, if the granularity under consideration is the portfolios, diversity was low. Portfolios were all very similar. The market eventually crashed in 2008. Recognizing such problems of granularity could play an important role across disciplines. Different granularities are also studied in ecology, there known as $\alpha$ and $\beta$ granularities (Schweiger and Laliberte 2022). In addition, the diversification cycles that we discussed earlier in the article are explained with causal models that link categories and subcategories of things at different granularities (Baciu 2018, 2019, 2021d).

In step 3.3, we proposed applying Simpson's diversity index to rank the level of diversity in a given urban area, and this index has already been applied in many other fields of science, as just described. It would seem relevant that scientists discuss diversity indices together with their merits and pitfalls across scientific boundaries. In a paper written more recently, we have proposed a new diversity index, the general diversity index, partly based on interdisciplinary considerations (Fig. 11; Baciu 2021d). 

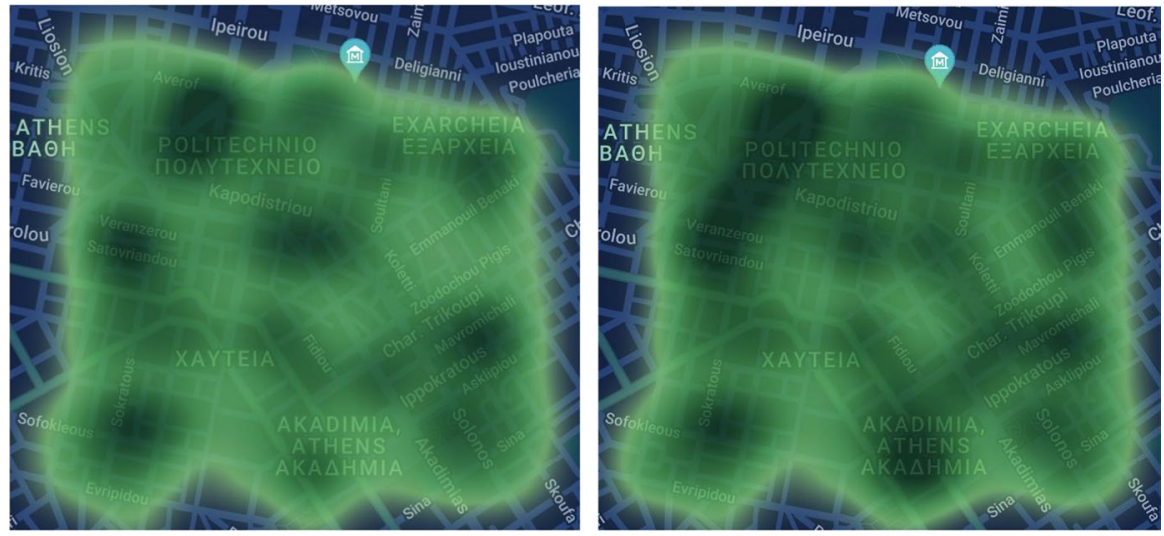

Fig. 11 The general diversity index allows us to weigh how much each type of urban activity contributes to urban diversity. The two maps shown in this figure represent two different sets of results with different weighing factors as applied to evaluating diversity in Athens, in a neighborhood near Omonoia Square (Nazou 2022). The same weighing can be done with the general diversity index in any other discipline. Weighing factors can be chosen to reflect known causal processes

In step 4, we created maps of diversity, yet such maps are also known from ecology, genetics, and human geography. The qualitative and quantitative interpretation of such maps should be relevant to all scientists, regardless of their discipline. We have also created a first map of diversification in Fig. 7. Such mapping could also be performed in other sciences.

In step 5, we validated our results through historical evaluation, and this is also done in island biogeography (MacArthur and Wilson 1967; Wilson 1999). Validation methods such as historical evaluation could be discussed very broadly across sciences. In addition, even before validation is performed, the robustness of the results can be checked. To check robustness, it is also advisable to go through several steps, which apply across multiple disciplines as recent research indicates (Nuijten 2022).

In step 6, we discussed the possibility that urban environments go through cycles of growth and diversification, and such cycles are already known in the humanities (Baciu 2020a, b). It should be relevant that a general framework for causal modeling is developed that unites all sciences in which cycles of growth and diversification are observed. Such a framework is developed in a subsequent article (Baciu 2021c).

These parallels across disciplines certainly mean that scientists could better pool their methods and theories by specifying which of the six steps their tools, techniques, and theories pertain to. Once a scientist specifies which step a tool is meant to perform, another scientist in a different field may find interest. Figuratively, our six steps are wormholes in the disciplinary material of scientific inquiry. 


\section{Discussion}

We have developed a method to map the diversity of urban activities, and we have demonstrated its application in multiple urban areas (Delfshaven, Sassi, Amsterdam, Athens). Through this method, it is possible to locate the most diverse centers of urban activity, a result that we validated through historical evaluation.

Yet, our method is more than a tool for analysis of existing conditions. We went on to show that architects and urban planners can utilize our method toward the assessment of new design proposals. Through this approach, practitioners and policy makers can go beyond locating some type of diversity; they can also choose to build buildings that enhance existing conditions. To demonstrate how one can facilitate such work, we have developed a first map that identifies how diversity changes, and we called it a map of diversification (Amsterdam).

The method that we developed is not a standalone. Mapping urban diversity was accompanied in this article by the mapping of diversity in architectural styles as well as by the mapping of cultural diversity in urban space. In addition, our method is compatible with methods utilized in ecology, soil science, and other disciplines. This insight led us to formulate six steps for the evaluation and mapping of diversity. These steps are intended to help researchers pool their tools and theories. We have discussed concrete situations where pooling of methods should be relevant. Our proposed steps could support a broad array of interdisciplinary exchange.

Finally, this article opens a new research direction into the dynamics of diversification. Next to developing and demonstrating the workings of a first method to map urban diversity, and next to formulating an interdisciplinary framework for the evaluation of diversity, the article also is a call for researchers to join forces with us in studying diversification in as many natural and cultural settings as possible. Creating diversity maps comes at almost no cost and at a potentially high benefit. All scientists should create some.

We are not standing still. In subsequent articles, we have meanwhile reconfirmed the applicability of our mapping method to other contexts where we validated the mapping results with additional validation techniques (Baciu and Della Pietra 2021b). We have also proposed ways in which our mapping method can be used to manage urban environments, for example through an early-warning system that can be activated to alert planners if diversity decreases or growth stagnates. And we have developed a causal framework to model the dynamics of diversification (Baciu 2021d).

\section{Summary of new contributions first presented in this article}

This present article was first made available as preprint in January 2021 and it has been updated in May 2022. The article makes original contributions in four distinct categories: (1) diversity evaluation, (2) simulation of diversification, (3) development of theory for the study of the dynamics of diversification, and (4) practical applications. The contributions are listed below. 
Diversity evaluation: We developed and applied a first method to map urban diversity. By urban diversity, we mean the diversity of activities that people engage in urban space. We proposed that our mapping method can be applied at any resolution, fine-grained or course-grained, and at any scale with local, regional, or global coverage. We applied our method to create diversity maps of architectural style with national coverage and diversity maps of culture with global coverage. By cultural diversity, we mean the diversity of ideas that are shared through public or social media. We broadened our approach and developed a first unified framework for the evaluation and mapping of diversity that can be applied across all sciences.

Simulation of diversification processes: We created the first map of diversification by simulating and mapping how hypothetical changes in the urban environment may cause changes in urban diversity.

Development of diversification theory: We defined the terms positive and negative diversification. We proposed that urban environments go through cycles of diversification that can be modeled with equations that we have previously used to model cultural diversification and that can be used to model cycles of diversification in almost any other field.

Practical applications: We have proposed the development of an early-warning system to help stabilize detrimental cycles of diversification in urban space. We suggested that diversification maps such as the one showed in Fig. 7 may be used as a tool for urban development serving developers, planners, as well as communities.

\section{Replicate the results}

All data and instructions necessary for replication of the results given in Figs. 3, 4, $5,6,8$ (Urban diversity maps) are given in a step-by-step manner in illustrations 2-8 and in Table 1. The text explains how to proceed, too. The data processing is done without code. The technical terms "granularity," "scale," "resolution," and "coverage" are explained in Fig. 12.

Data to reproduce the results given in Figs. 2 and 3 (Diversity map for Delfshaven) are found at maps.google.com. The Google Streetview version is specified in Data Accessibility. Both Fig. 1 and the text explain how to proceed. The data processing is done without code.

Data to reproduce Fig. 7 (Diversification map for Amsterdam) was originally published by the city of Amsterdam and is now available in our online repository https:// doi.org/10.17605/OSF.IO/NWKPZ. Code to process is available upon request from github.com/dan-baciu The estimate is obtained as follows: use Amsterdam data for urban functions, choose granularity $1 \mathrm{~m}^{2}$, overlay with 0.001 degree grid, compute Simpson's index, simulate an increase of factor 1.5, perform the same analysis, subtract the first set of result from the second, and map all negative values (negative diversification) as heat map.

Data to reproduce Fig. 9 (Diversity in Sassi di Matera) together with step-by-step instructions are given in Baciu and Della Pietra 2021b.

Data to reproduce Fig. 10 (News Diversity Map) are found in our previous article, Baciu 2020a, b, see Data Accessibility. Each map in Baciu 2020a, b Fig. 2, 


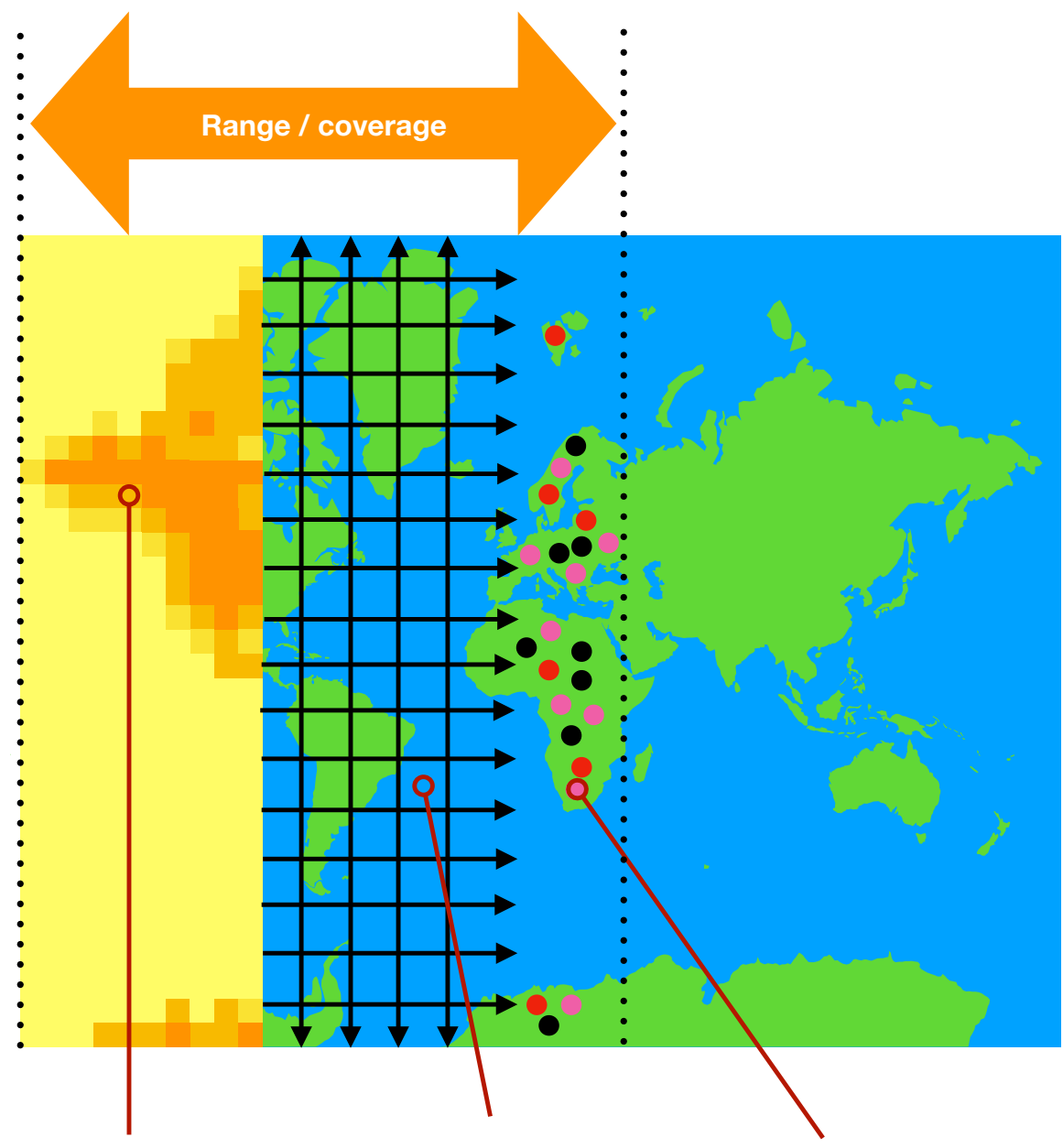

Resolution Scale

Granularity

Fig. 12 Some geographic parameters in diversity mapping. First, the data that are evaluated have a given "granularity." The granularity mostly is given by the data; it is the amount of detail captured when the data are collected. Second, the data are evaluated with an analysis grid or something the like. In our initial example, we chose to consider diversity in square urban areas of $100 \mathrm{~m} \times 100 \mathrm{~m}$. There are many options in defining these areas: the squares could also be circles, or their borders could be fuzzy, etc. Overall, the analysis grid can be more narrow-meshed or more broad-meshed, which we interpret as the "scale" of the analysis. Third, the number of points or the distance between points on the map at which diversity is estimated makes the resolution of the analysis. In our example, each yellow square is a point at which diversity is estimated independent of other points. Finally, each analysis has a certain range that it covers

interactive version, is considered a species in the diversity index. Calculate Simpson Diversity Index $1 / \mathrm{D}$. Grid size $1^{\circ}$. Code to automatize the procedure is available upon publication from github.com/dan-baciu. 
Table 1 Categories of urban activities considered in the Delfshaven area

\begin{tabular}{|c|c|c|}
\hline $\begin{array}{c}\text { Activity } \\
\text { categories }\end{array}$ & Color & Category description \\
\hline Home & & $\begin{array}{c}\text { Terrace, Semi-Detached, Detached, Tower } \\
\text { Blocks, Apurtments ate. }\end{array}$ \\
\hline Office & & 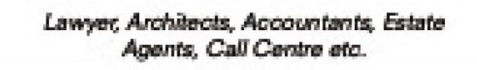 \\
\hline Religion & & Church, Mosque, Shasogowe, Tomplos ate. \\
\hline Education & & 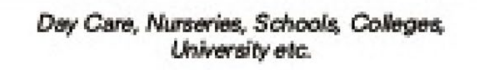 \\
\hline Health & & 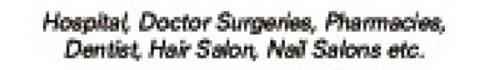 \\
\hline Restaurant & & $\begin{array}{c}\text { AV tyos of sit down and takesway las lood } \\
\text { shops. }\end{array}$ \\
\hline Shopping & & 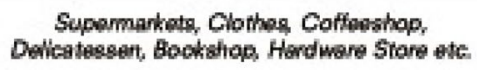 \\
\hline Leisure time & & 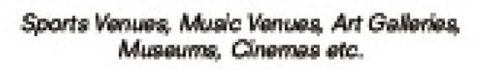 \\
\hline Public transp: : & & $\begin{array}{c}\text { Matro Statian, Tram Station, Whiter Supplies, } \\
\text { Wentlation ate. }\end{array}$ \\
\hline Renewal & & Stan-fronts that wo currently unused. \\
\hline
\end{tabular}

The system of categorization (or taxonomy) may be chosen to reflect natural clusters, or it may simply be an intuitive experimenter's choice, as it is in this example. However, once the analysis is performed, it is relevant to test whether the results are good estimates. We did this step through historical evaluation

Data to reproduce Fig. 11 (Athens diversity maps) are available on request. Code to automatize the procedure is available upon publication from github.com/ dan-baciu.

Author contributions DCB, as an assistant professor, wrote the article, contributed the research concept, developed the six steps toward diversity mapping together the unified framework for diversity mapping, created the smaller part of diversity maps and visuals, and instructed DM, CB, DDP, LVL, and AN. Duola Mi, as a bachelor student under DCB: supervision at University of California Santa Barbara in 2019-2020, collaborated on creating diversity maps of architectural style in the United States. CB, as a master student under DCB: supervision at TU Delft in 2020-2021, reviewed literature, collected and processed the Google data for Delfshaven, developed the classification system for urban diversity as shown in Table 1, chose red-yellow color schemes, and created the diversity maps and visuals for Delfshaven. DDP, as master student under DCB: supervision at TU Delft in 2020-2021, collaborated equally to DCB toward the study of diversification in Sassi di Matera, Italy, in particular collecting all data, performing the analysis, and creating the diversity maps for Sassi. LVL, as a master student under DCB: supervision at TU Delft in 2021-2022, studied Amsterdam, locating the data used for the diversification map of Amsterdam. Anna Nazou, as a master student under DCB: supervision in 2021-2022, collected data and developed the classification system for Athens. 
Data availability Urban diversity map (Figs. 3, 4, 5, 6, 9): Google maps and Google street view recorded Nov. 2020. Data publicly available at maps.google.com News diversity map (Fig. 10, above): Baciu DC (2020). "Cultural life: Theory and empirical testing." Biosystems 197.104208. Figure 2. Article publicly available at: https://doi.org/10.1016/j.biosystems.2020.104208 Data visualized and publicly available at: https://doi.org/10.25496/W2RP4H. Diversity of architectural styles in the United States (Fig. 10, below): Data from SAHARA, 2019. Dataset available on author's project page https://doi.org/10.17605/OSF.IO/ NWKPZ

Open Access This article is licensed under a Creative Commons Attribution 4.0 International License, which permits use, sharing, adaptation, distribution and reproduction in any medium or format, as long as you give appropriate credit to the original author(s) and the source, provide a link to the Creative Commons licence, and indicate if changes were made. The images or other third party material in this article are included in the article's Creative Commons licence, unless indicated otherwise in a credit line to the material. If material is not included in the article's Creative Commons licence and your intended use is not permitted by statutory regulation or exceeds the permitted use, you will need to obtain permission directly from the copyright holder. To view a copy of this licence, visit http://creativecommons.org/licen ses/by/4.0/.

\section{References}

Amundson R (2021) Factors of soil formation in the 21st century. Geoderma 391:114960

Baciu DC (2015) Systemic cooperation: scientists, architects, urban space and tourism. JETK 4, pp 27-30. https://escholarship.org/uc/item/81h50276

Baciu DC (2018) From everything called Chicago school towards the theory of varieties. Doctoral dissertation, Illinois Institute of Technology. http://hdl.handle.net/10560/4376

Baciu DC (2019) The Chicago school: large-scale dissemination and reception. Prometheus 2:2041 https://escholarship.org/uc/item/22v9g5mn

Baciu DC (2020a) Cultural life: theory and empirical testing. BioSystems 197:104208 DOI: https://doi. org/10.1016/j.biosystems.2020.104208

Baciu DC (2020b) Cultural diversification processes and the culture radar. MAT 2019-2020 lecture series, April 20, 2020. https://escholarship.org/uc/item/4993b2vq

Baciu DC (2021a) Creativity and diversification: What digital systems teach. Thinking Skills and Creativity. https://doi.org/10.1016/j.tsc.2021.100885

Baciu DC (2021b) Questions and a myriad answers: coming together and drifting apart in the historical sciences. In: Bruno Zevi. Franco Angeli, Milan. https://doi.org/10.31219/osf.io/g8d9c

Baciu DC (2021c) 10 Causal Models for Life. OSF preprints DOI: https://doi.org/10.31219/osf.io/8uyc3

Baciu DC (2021d) $\varphi$ is for Causality, + is for creativity, $\times$ is for diversity. OSF preprints. https://doi.org/ $10.31219 /$ osf.io/byndg

Baciu DC, Cellucci V (2022) Paths of wind and of research. Contribution to exhibition. Atomic reactions. TU Delft Library. https://doi.org/10.31219/osf.io/h4g6p

Baciu DC, Kahnt N (2015) Tour Eiffel: A review combined with a big data analysis. Phoenix, March 2015:56-61. https://escholarship.org/uc/item/51f3d4bz

Baciu DC, Della Pietra D (2021a) Cycles of Diversification in Urban Environments. OSF preprints DOI: https://doi.org/10.31219/osf.io/f43xh

Baciu DC, Della Pietra D (2021b) Cicluri de diversificare în spațiul urban. Igloo 2021. https://escholarsh ip.org/content/qt7cb3s2f9/qt7cb3s2f9.pdf

Baciu DC, Mi D, Bichall C, Della Pietra D, Loevezijn L, Nazou A (2021) Mapping diversity: from ecology and human geography to urbanism and culture. [preprint of this article]. https://doi.org/10. 31219/osf.io/sdvaz

Baciu DC (2022) Mapping Urban Diversity: Applications. OSF preprints DOI: https://doi.org/10.31219/ osf.io/buajg

Birchall C (2021) Cultivating spatial diversity: the Nieuwe Binneweg Forum. TU Delft. http://resolver. tudelft.nl/uuid:5bee1ecd-b474-4e2e-82f4-6962502dfdfe

Della Pietra D (2021) Mapping urban diversity: vernacular, modernist and contemporary Matera. TU Delft. http://resolver.tudelft.nl/uuid:159d59b9-f44b-465c-8e5b-8859bd0e0b37 
Dijkstra L, H Poelman (2012) Cities in Europe: the new OECD-EC definition regional focus 01/2012

Dupertuis Bangs J (2020) New light on the old colony: Plymouth, the Dutch context of toleration, and patterns of pilgrim commemoration. Brill, Leiden

Raszka P, Baciu DC (2022) Mapping Commuter Diversity in New York City: Diversity and vertical growth may form positive feedback loop. OSF preprints DOI: https://doi.org/10.31219/osf.io/etn32

Fisher RA, Corbet AS, Williams CB (1943) The relation between the number of species and the number of individuals in a random sample of an animal population. J Animal Ecol 12:42

Friss E (2019) On Bicycles: a 200 year history of cycling in New York City. Columbia University Press, New York

Gould W (2000) Remote sensing of vegetation, plant species richness, and regional biodiversity hotspots. Ecol Appl 10:1861-1870

Guo Y, Gong P, Amundson R (2003) Pedodiversity in the United States of America. Geoderma 117:99-115

Hirschman AO (1945) National power and the structure of foreign trade. University of California Press, Berkeley

Ibanańez JJ, De-Albs S, Bermúdez FF, García-Álvarez A (1995) Pedodiversity: concepts and measures. CATENA 24:215-232. https://doi.org/10.1016/0341-8162(95)00028-Q

Jacobs J (1961) The death and life of great American cities. Random House, New York

Jenkins CN, Pimm LS, Joppa LN (2013) Global patterns of terrestrial vertebrate diversity and conservation. Proc Natl Acad Sci USA 110:11457-11462

Loevezijn L (2022) Thesis, TU Delft http://resolver.tudelft.nl/uuid:9bcc1b78-89a7-4ba3-a5d5-e9265 $721 \mathrm{e} 00 \mathrm{~d}$

Lynch K (1960) The Image of the City. MIT Press, Boston

MacArthur RH, Wilson EO (1967) The theory of island biogeography. Princeton University Press, Princeton

Maunier R (1910) The Definition of the City. Am J Sociol 15:536-548

Mintzker Y (2012) The Defortification of the German city 1689-1866. Cambridge University Press, Cambridge

Miraldo A, Li S, Borregaard MK, Flórez-Rodríguez A, Gopalakrishnan S, Rizvanovic M, Wang Z, Rahbek C, Marske KA, Nogués-Bravo D (2016) An Anthropocene map of genetic diversity. Science 353(6307):1532-1535. https://doi.org/10.1126/science.aaf4381

Nazou A (2022) Thesis. TU Delft http://resolver.tudelft.nl/uuid:e6dba926-b05c-4152-8e4f-7759d6a61bc5

Nowak MA, May RM (2000) Virus Dynamics. Oxford University Press, Oxford

Nowak MA, Anderson RM, McLean AR, Wolfs TFW, Goudsmit J, May RM (1991) Antigenic diversity thresholds and the development of AIDS. Science 254:963-969

Nuijten MB. "Checking Robustness in 4 Steps". Presentation, Tilburg University. www.metascience.com. Accessed 16 May 2022

Pritchard JK, Stephens M, Donnelly P (2000) Inference of population structure using multilocus genotype data. Genetics 155:945-959

Raszka P (2022) Thesis. TU Delft. https://doi.org/10.4233/uuid:206fd84d-a011-4c75-ae6f-45d864d8b7 $1 \mathrm{e}$

Sawyer JD (1925) The romantic and fascinating story of the pilgrims and puritans: a complete and authentic narrative of their voyages, perils and adventures, their beginnings and progress, their activities and their times. H.S. Nichols, New York

Schweiger AK, Laliberté E (2022) Plant beta-diversity across biomes captured by imaging spectroscopy. Nat Commun 13:2767

Simmel G (1903) Die Großstädte und das Geistesleben. Die Großstadt: Vorträge und Aufsätze zur Städteausstellung. Gehe-Stiftung, Dresden, pp 185-206

Simpson EH (1949) Measurement of diversity. Nature 163(4148):688-688. https://doi.org/10.1038/16368 $8 \mathrm{a} 0$

United Nations Climate Change (2021) Economic diversification. https://Unfccc.int. Accessed 21 Jan 2021

Vertovec S, Hiebert D, Gamlen A, Spoonley P (2021) Superdiversity: Today's Migration has Made Cities More Diverse Than Ever in Multiple Ways. https://Mpg.de. Accessed 21 Feb 2021

Wilson EO (1999) The Diversity of Life. W.W. Norton, New York

Wurm S (1996) Atlas of the World's Languages in Danger of Disappearing. UNESCO Publishing, Paris

Yule U (1944) Statistical Study of Literary Vocabulary. Cambridge University Press, Cambridge 